\title{
Turismo 2.0 como herramienta para promocionar los atractivos culturales de Guayaquil
}

\section{Tourism 2.0 as a tool to promote the cultural attractions of Guayaquil}

\author{
Ana Luisa López Brunett \\ Universidad Internacional del Ecuador, Ecuador \\ Ericka Verdesoto Masache \\ Universidad Internacional del Ecuador, Ecuador \\ Antonio Humberto López Brunett \\ Universidad Politécnica Salesiana, Ecuador
}

Autor por Correspondencia: anlopezbr@uide.edu.ec, everdesotoma@uide.edu.ec, alopezb1@est.ups.edu.ec

Fecha de recepción: 6 de Marzo de 2017 - Fecha de aceptación: 5 de Junio de 2017

\section{Resumen}

La sociedad está atravesando por un desarrollo que implica la utilización de nuevas tecnologías, las cuales en conjunto podrían ayudar a evolucionar a los diferentes sectores económicos, considerando entre ellos y de manera particular, al productivo y a todo lo que concierne al ámbito turístico. A partir de estos argumentos y cuando se busca comprender cuál es el papel que ocupa la tecnología como una parte intrínseca del sector antes mencionado, cabe destacar entonces la convergencia que existe entre lo digital y lo que implica el proceso de globalización, como un aspecto culminante para lograr dentro de este marco una revolución de la información y de las implicaciones que esto conlleva para lograr una verdadera transformación del turismo. (Becerra, Melo, \& Rocha, 2012, párr. 6)

Palabras claves: web 2.0; turista digital; turismo; turista 2.0

\begin{abstract}
Society is going through a development that implies the use of new technologies and as a whole could help in the evolution of different economy areas among them and most particularly the productive area and all that concerns the tourism industry. Starting from these arguments and when we look to understand the importance of technology as an intrinsic part of the sector mentioned before, is good to highlight the convergence that exists between the digital world and what implies a globalization process as a culminant aspect to achieve inside this parameter a revolution in the tourism transformation.
\end{abstract}

Key words: web 2.0; Digital tourist; tourism; tourist 2.0 


\section{Introducción}

Los precedentes sobre la actividad turística no tienen una fecha precisa de iniciación en lo que respecta a su historia; pero se hace mención a las razones por las que los individuos comenzaron a trasladarse a los diferentes lugares de su habitual sitio de origen, siendo una de ellas la búsqueda de medicamentos, curas de enfermedades, peregrinaciones religiosas o actividades relacionados con el comercio. Al realizar cada una de estas labores, los individuos buscaban lugares donde pernoctar durante el período fuera de su residencia y es aquí donde recurrían a monasterios, establos o las denominadas posadas que con los años adaptaron el nombre de "Hotel", término francés que significa casa adosada. (Perrot, 2011).

En la actualidad la sociedad está atravesando por un enorme desarrollo que implica la utilización de nuevas tecnologías, las cuales en conjunto podrían ayudar a la evolución de los diferentes sectores económicos, considerando entre ellos y de manera particular, al productivo y a todo lo que concierne al ámbito turístico. A partir de estos argumentos y cuando se busca comprender cuál es el papel que ocupa la tecnología como una parte intrínseca del sector antes mencionado, destacamos la convergencia que existe entre lo digital y lo que implica el proceso de globalización, como un aspecto culminante para lograr dentro de este marco una revolución de la información y de las implicaciones que esto conlleva para lograr una verdadera transformación del turismo. (Becerra, Melo, \& Rocha, 2012, párr. 6).

En esta misma línea y dirección sobre el uso de las nuevas tecnologías, han emergido los "turistas digitales", el cual es conocido como aquel que utiliza todas las herramientas disponibles en su teléfono inteligente para lograr a través de su uso, hacer cosas o actividades que años atrás llevaba más tiempo y dinero realizar, como por ejemplo la reservación de hoteles, boletos aéreos, obtener asesoría turística y en términos generales, el llevar a cabo la planificación u organización de su viaje.

Frente a este tipo de turistas, se hace necesario tener un mayor grado de conocimiento acerca de las necesidades y gustos de los usuarios, con la necesidad de implementar ideas, productos y servicios innovadores que se acoplen a las continuas y actuales demandas del mercado. Asegurar el éxito con esta clase de servicios no es una tarea fácil o sencilla para las empresas del sector, mucho más si la sociedad no está comprometida a ser partícipe de este proceso, que contempla en toda su extensión una mejora de la propuesta turística que ofrece una ciudad.

\section{La Tecnología aplicada al Turismo}

Durante los últimos años, la ciencia de la tecnología en información y comunicación, también conocida como "Tics", ha logrado desarrollar una serie de atributos que contribuyen en todo su contexto beneficios para los sectores productivos de la sociedad. Es habitual notar la presencia de la tecnología en las diversas actividades diarias, debido fundamentalmente al ininterrumpido prolongamiento de todo su proceso, que de acuerdo al desenvolvimiento de la oferta y demanda turística, requiere cada vez más de nuevos y mejores productos-servicios que puedan satisfacer las demandas y exigencias de los turistas.(Guevara \& Cerezo , 2015). 
Como complemento a lo anterior, con la aplicación de la investigación tecnológica aplicada al sector turístico tenemos la definición de Turismo 2.0, que entre varias conceptualizaciones se lo ha determinado como una "herramienta basada en un nuevo modelo de interacción virtual o de sociedad en red, donde los turistas, viajeros y profesionales del sector turístico, interactúan entre sí aportando opiniones y experiencias personales.” (Pérez, 2016, párr. 2). En otras palabras y frente a la evolución de los mercados, los destinos en general requieren de herramientas que asistan a sus visitantes, para que estos sirvan como instrumentos que puedan dar a conocer no sólo aspectos publicitarios sino todo lo que implique la promoción de los diversos atractivos de las zonas. (Pérez, 2016). Los destinos persiguen la innovación y el valor agregado que los diferencian de sus competidores ya que el viajero actual y potencial tiene un número mayor de exigencias y expectativas, que está actualizado y además, informado sobre las últimas tendencias tecnológicas.

\section{Tendencias de Consumo}

Las tendencias de consumo sobre la descarga de aplicaciones móviles es un tema que se encuentra en incremento en un mundo globalizado que no piensa detenerse y que cada vez se vuelve más dinámico e interactivo, las app son consideradas como herramientas para realizar actividades cotidianas que facilitan la vida de los consumidores. En el mercado mundial existen aplicaciones móviles para satisfacer necesidades, gustos y preferencias de los usuarios, según el listado de las 15 APPS más utilizadas en el mundo (Forbes, 2013), la aplicación Google Map es una de las herramientas más descargadas seguidas por Facebook mobile apps.

Para los años 2011 y 2012 las aplicaciones móviles lograron descargas de 23.000 a 32.000 millones respectivamente, llegando a ser iOS y Android los sistemas operativos que dominan el mercado de los Smartphone, seguidos de Symbian y Windows Phone. Desde el sistema operativo de los móviles de iOS se alcanzó para el 2013 un total de 40.000 millones de descargas, mientras que Android obtuvo 10.000 millones. Para las aplicaciones turísticas el número no se detiene, ya que entre las más demandadas están los mapas y guías, liderando Google Maps y Google Earthcon 50 y 31 millones cada una.

Por otro lado, las aplicaciones de boletos aéreos tienen una participación del 25\% de las apps más descargadas, entretanto tripadvisor dentro de la categoría de hoteles abarca el mercado con un $27 \%$. De la misma manera de acuerdo a datos obtenidos, las aplicaciones gratuitas más descargadas corresponden a redes sociales y recomendadores. (Sociedad Estatal SEGITTUR, 2013)

En Ecuador y de acuerdo a la información publicada por el Instituto Nacional de Estadísticas y Censos, los ecuatorianos forman parte de quienes hacen uso frecuente e indispensable de teléfonos inteligentes. El 16,9\% (1'261.944) de las personas de cinco años y más que tienen celular poseen un teléfono inteligente (Smartphone), lo que representa un crecimiento de $141 \%$ frente al 2011, según los últimos datos de la Encuesta de Tecnologías de la Información y la Comunicación (TIC) del Instituto Nacional de Estadística y Censos (INEC). 
El estudio, que se realizó en diciembre de 2013, se hizo en 21.768 hogares a personas de 5 años y más, a nivel nacional, regional, provincial, de nivel urbano y rural. (Instituto Nacional de Estadísticas y Censos, 2014).

\section{Tipo de investigación}

"Turismo 2.0 como herramienta para promocionar los atractivos culturales de Guayaquil" es una investigación del tipo descriptiva porque tiene como finalidad detallar de manera más específica las características importantes y establecer lo que considera el mercado Guayaquileño en referencia al producto - servicio de apps para pomocionar los atractivos culturales de Guayaquil. Para dicha investigación se efectuará un compendio de información indispensable de una muestra de la población seleccionada. (Merino, Pintado, Sánchez, \& Grande, 2015).

\section{Resultados de las Encuestas}

Se puede observar que el $62 \%$ de los encuestados posee servicio de pre-pago y el $38 \%$ cancela un servicio de post pago mensualmente.

\section{TIPO DE SERVICIO QUE POSEE}

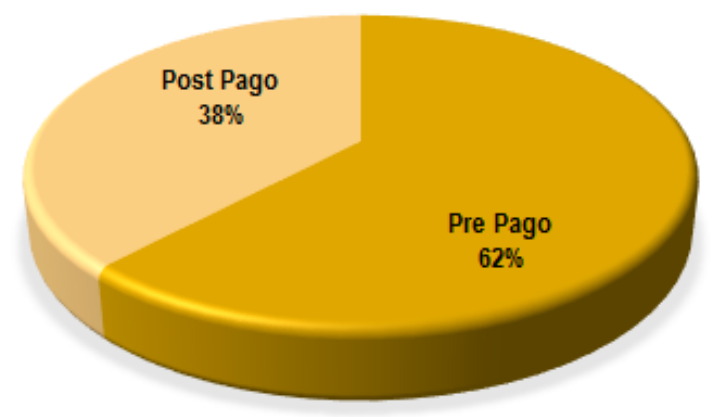

Gráfica 2: tipo de servicio telefónico

Fuente: Investigación de mercado, 2016

Elaborado por: ErickaVerdesoto M.

El 54,67\% de las personas encuestadas respondieron que es muy frecuente la regularidad con la que visitan los sitios turísticos y culturales de Guayaquil. El 36, 67\% lo hace poco frecuente, y el 8,67\% nada frecuente. 
4. ESCOJA LA REGULARIDAD CON LA QUE USTED VISITA

LOS SITIOS TURISTIICOS Y CULTURALES DE GUAYAQUIL

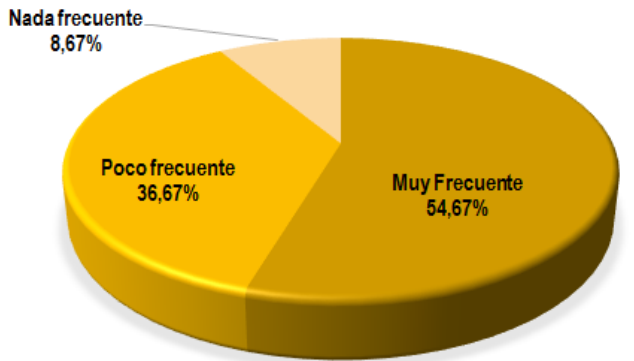

Gráfica 4: Regularidad con las que visita los sitios turísticos y culturales de Guayaquil.

Fuente: Investigación de mercado, 2016 Elaborado por: ErickaVerdesoto $\mathrm{M}$.

Del total de los encuestados el 59,33\% afirmó que descarga aplicaciones móviles muy frecuentemente, el $26 \%$ poco frecuente y el $14,67 \%$ nada frecuente.

\section{CON QUÉ FRECUENCIA DESCARGA APLICACIONES MÓVILES EN SU TELÉFONO CELULAR?}

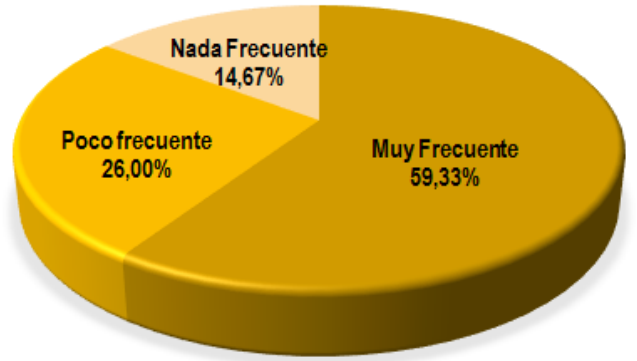

Grafica 5: Frecuencia para descargar aplicaciones móviles en su celular.Fuente: Investigación de mercado, 2016

Elaborado por: ErickaVerdesoto

Los segmentos más importantes representa el 37,33\% descarga aplicaciones, el 19\% juegos, el $12 \%$ música - audio, el $10 \%$ deporte, $6 \%$ estilo de vida y en menores porcentajes están salud y bienestar, transporte, educación, otros.

\section{LA CATEGORÍA DE LAS APLICACIONES QUE MÁS} DESCARGA SE RELACIONAN CON...

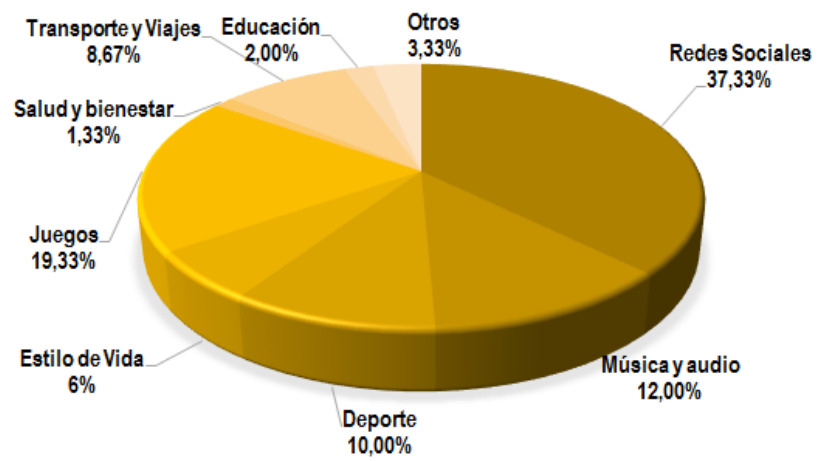

Grafica 6: Categoría de las aplicaciones con más descargas. 
De los dos únicos segmentos posibles, el 72,67\% afirmó que las aplicaciones que descarga son gratuitas y el 27,33\% aseguró que las aplicaciones que adquiere a través de la telefonía móvil son pagadas.

\section{LAS APLICACIONES QUE USTED DESCARGA \\ SON..}

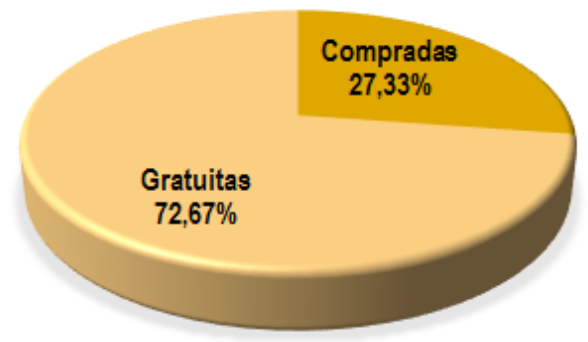

Grafica 7: Tipos de aplicaciones

Fuente: Investigación de mercado, 2016

Elaborado por: ErickaVerdesoto

El 35\% de los encuestados no ha descargado aplicaciones que estén relacionadas con la ciudad, el 56\% prefirió no contestar a esta pregunta y apenas el 8\% respondió que sí lo había hecho en ocasiones anteriores.
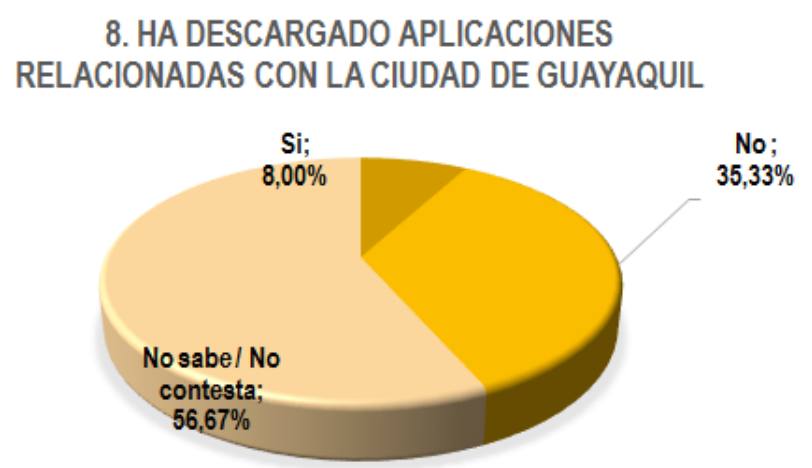

\section{Grafica 8: Descargas de aplicaciones móviles relacionadas con la ciudad de Guayaquil \\ Fuente: Investigación de mercado, 2016 \\ Elaborado por: ErickaVerdesoto}

El 70\% de los encuestados afirmó que sí le gustaría que existiera una aplicación de los sitios turísticos y culturales más importantes de la ciudad de Guayaquil. El 9,33\% respondió que no y el 20,67\% no respondió a esta proposición. 


\section{LE GUSTARÍA QUE EXISTIERA UNA APLICACIÓN CON INFORMACIÓN DE LOS SITIOS TURISTICOS Y CULTURALES \\ DE GUAYAQUIL}

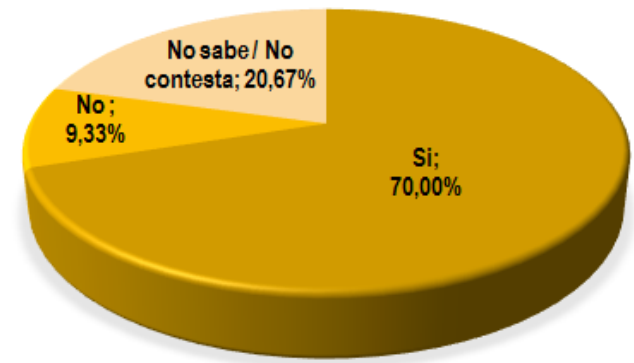

Grafica 9: Le gustaría que existiera una aplicación con información de los sitios turísticos y culturales de Guayaquil

Fuente: Investigación de mercado, 2016

Elaborado por: ErickaVerdesoto

El 32\% de los encuestados le gustaría que la aplicación tuviera información sobre sitios de entretenimiento, al 30\% sobre restaurantes, al 14\% sobre hoteles y alojamientos, al 10.67\% sobre teatros, al $8 \%$ acerca de lugares de compra y finalmente al 5,33\% sobre agencias de viajes y turismo.

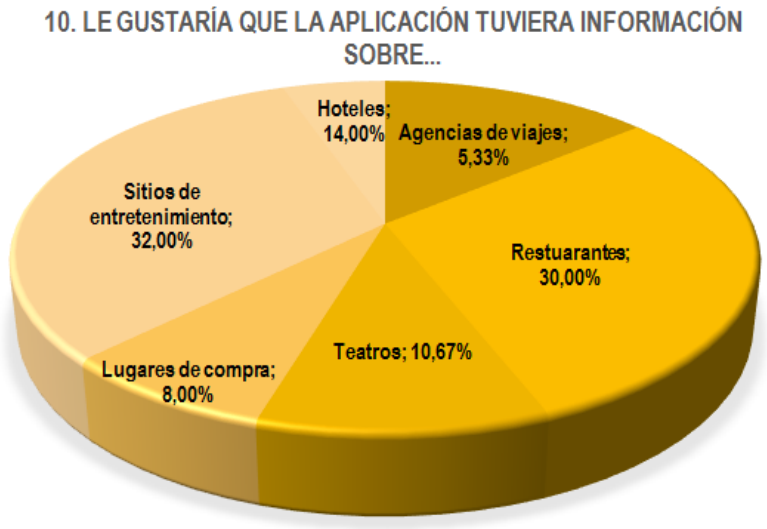

Grafica 10: Información que le gustaría que tuviera la aplicación móvil.

Fuente: Investigación de mercado, 2016

Elaborado por: ErickaVerdesoto

\section{Conclusiones}

De las encuestas realizadas se pudo determinar algunas características de los usuarios del servicio de telefonía móvil en la que prevalecen los segmentos de personas jóvenes cuyas edades fluctúan entre los 20 y 39 años. De todos los grupos el que mayor incidencia generó fue de las personas o usuarios cuyas edades estaban entre los 25 y 29 años de edad. Una característica importante se remarcó entre aquellos que aún siguen siendo mayoría, es decir, los que mantienen un servicio pre - pago para tener comunicación con otras personas. 
De todos los encuestados, el $60 \%$ afirmó conocer los diferentes sitios turísticos y culturales de Guayaquil; pero la regularidad de vistas se reduce drásticamente entre quienes afirmaron sí conocerlos ya sea en su totalidad o de manera parcial. El 59\% de las personas afirmó que descargan aplicaciones móviles con mucha frecuencia y entre las categorías que más prevalecen están las redes sociales, los juegos, música - audio, y transporte. Como complemento a lo anterior, poco es el número de personas que se enfocan en descargar aplicaciones cuyas categorías se centran más en educación, salud - bienestar, estilo de vida, entre otros. El $72 \%$ de los encuestados se inclina o prefiere descargar aplicaciones móviles que son gratuitas. Esto se debe por lo regular a que también la mayoría de los encuestados tienen el servicio pre - pago.

Con respecto a la ciudad de Guayaquil, escaso es el número de personas que han descargado aplicaciones que están relacionadas directamente con sitios o lugares referenciales de la ciudad. No obstante, al 70\% sí le gustaría que de existir una aplicación móvil con las características deseadas, ésta pudiera ser utilizada como complemento o indicador para la realización de ciertas actividades.

Como resultado de la investigación se logra reconfirmar que los avances tecnológicos enfocados a desarrolladores de herramientas cuentan con un prolongado progreso, dando oportunidades de ofertar mejores y nuevos servicios en el mercado de aplicaciones móviles, conforme a esto la demanda se vuelve exigente y requiere de más opciones en dicha industria, generando de esta forma ingresos considerables a quien diseñe apps. Dentro del plano turístico, esta industria está obteniendo ventajas, aportando así a la promoción turística de algunos destinos o de establecimientos relacionados con los mismos. Cabe mencionar que en los productos servicios tecnológicos siempre es indispensable que se realicen las respectivas actualizaciones de los programas y/o plataformas.

En el caso de Guayaquil, los desarrolladores de aplicaciones móviles para teléfonos inteligentes se presentan con propuestas innovadoras en función del diseño y estrategias de promoción generando ideas atractivas para los turistas locales y /o extranjeros que pueden utilizar la plataforma como guía o agenda turística-cultural y cuya propuesta es contribuir para que la ciudad presente cambios y llegue a posicionarse en la mente de los turistas como una urbe que hace uso de las herramientas tecnológicas para ofrecer un valor agregado al turista.

\section{Bibliografía}

Becerra, É., Melo, M., \& Rocha, A. (Septiembre - Octubre de 2012). El turismo en la sociedad de la información. Un abordaje conceptual sobre el "pos-turismo". Estudios y perspectivas en turismo, XXI(5), párr. 6. Recuperado el 28 de Junio de 2016, de Disponible en: http://www.scielo.org.ar/scielo.php?script=sci_arttext\&pid=S1851-17322012000500011

Cabarcos, N. (2006). Promoción Y Venta de Servicios Turísticos. Vigo: Ideaspropias Editorial S.L.

Dalles, P. (23 de Octubre de 2012). Abc Color. Obtenido de Edición Impresa: El turismo y sus antecedentes históricos: Encontrado en: http://www.abc.com.py/edicionimpresa/suplementos/escolar/el-turismo-y-sus-antecedentes-historicos-468250.html 
Dvoskin, R. (2004). Fundamentos de marketing: teoría y experiencia. Buenos Aires: Ediciones Granica S.A.

Forbes. (12 de Agosto de 2013). Las 15 apps más utilizadas del mundo. Lista de empresas. Recuperado el 22 de Mayo de 2016, de Las 15 apps más utilizadas del mundo: http://www.forbes.com.mx/las-15-apps-mas-utilizadas-del-mundo/

Forbes. (12 de Agosto de 2013). Las 15 apps más utilizadas del mundo. Recuperado el 22 de Mayo de 2016, de Las 15 apps más utilizadas del mundo: http://www.forbes.com.mx/las-15-apps-masutilizadas-del-mundo/

Freund, J. E., \& Simon, G. A. (1994). Estadística Elemental (Primera ed.). (S. Conmy, Ed., \& P. H. S.A., Trad.) Naucalpan de Juárez, México: Cámara Nacional de la Industria.

Gómez, M. (2006). Introducción a la Metodología de la Investigación cientifíca. Córdoba: Ediciones Brujas.

Grasso, L. (2006). Encuestas. Elementos para su diseño y análisis. Córdoba: Ediciones Brujas.

Guevara, A., \& Cerezo, A. (2015). El papel estratégico de las tecnologías de. Málaga.

Icart, M., FuenteIsaz, C., \& Pulpón, A. (2006). Elaboración y presentación de un proyecto de investigación y una tesina. Barcelona: Edicions Universitat Barcelona.

Instituto Nacional de estadísticas y censos. (2010). Instituto Nacional de estadísticas y censos. Recuperado el 01 de 07 de 2016, de Población y Demografía: Obtenido de: http://www.ecuadorencifras.gob.ec/censo-de-poblacion-y-vivienda/

Instituto Nacional de Estadísticas y Censos. (16 de Mayo de 2014). 1,2 millones de ecuatorianos tienen un teléfono inteligente (Smartphone). Recuperado el 22 de Mayo de 2016, de 1,2 millones de ecuatorianos tienen un teléfono inteligente (Smartphone): http://www.ecuadorencifras.gob.ec/12-millones-de-ecuatorianos-tienen-un-telefonointeligente-smartphone/

Instituto Nacional de Estadísticas y Censos. (Marzo de 2016). Ecuador en cifras. Recuperado el 05 de Junio de 2016, de Reporte de Pobreza: Obtenido de: http://www.ecuadorencifras.gob.ec/documentos/webinec/POBREZA/2016/Marzo_2016/Informe\%20pobreza-mar16.pdf

Instituto Nacional de Estadísticas y Censos. (30 de Marzo de 2016). Encuesta Nacional de Empleo, desempleo y subempleo. Recuperado el 05 de Junio de 2016, de Encuesta Nacional de Empleo, desempleo y subempleo: Obtenido de: http://www.ecuadorencifras.gob.ec/documentos/webinec/EMPLEO/2016/Marzo-2016/Presentacion\%20Empleo_0316.pdf

Instituto Nacional de Estadísticas y Censos. (30 de Enero de 2016). Inflación Mensual 2016. Recuperado el 05 de Juni0 de 2016, de Inflación Mensual 2016: http://www.ecuadorencifras.gob.ec/documentos/webinec/Inflacion/2016/InflacionEnero2016/Reporte_inflacion_201601.pdf 
Kotler, P., \& Armstrong, G. (2008). Principios de Marketing (Duodécima ed.). Madrid: Pearson Educación. Recuperado el 24 de Agosto de 2016

Leiva, J. L. (2014). Realidad Aumentada bajo Tecnología Móvil. Málaga: Publicaciones y Divulgación Científica. Universidad de Málaga.

Merino, M., Pintado, T., Sánchez, J., \& Grande, I. (2015). Introducción a la investigación de mercados. Madrid: ESIC Editorial.

Ministerio de Turismo. (06 de Enero de 2016). El turismo dinamizó la economía ecuatoriana por USD 97 millones durante los dos últimos feriados. Recuperado el 05 de Junio de 2016, de El turismo dinamizó la economía ecuatoriana por USD 97 millones durante los dos últimos feriados: http://www.turismo.gob.ec/1-millon-187-mil-turistas-recorrieron-el-pais-durante-losferiados-de-navidad-y-ano-nuevo/

Monferrer, D. (2013). Fundamentos de Marketing (Primera ed.). Castelló de Plana, España: Publicacions de la Universitat Jaume I. Recuperado el 26 de Agosto de 2016

Morère, N., \& Perelló, S. (2013). Turismo Cultural: patrimonio, museos y empleabilidad. (E. Lizarralde, Ed.) Madrid, España: Fundación EOI. Recuperado el 2 de Agosto de 2016, de Disponible en: http://www.turismoculturalun.org.ar/pdfs/El_turismo_Cultural_Patrimonio_museos_y_emple abilidad.pdf

Pérez, M. (2016). Asesoramiento, venta y comercialización de productos turísticos. IC.

Perrot, M. (2011). Historia de las alcobas. Ediciones Siruela.

Plazas, F. (2011). Investigación de Mercado (Primera ed.). Bogotá, Colombia: Ediciones de la U. Recuperado el 28 de Octubre de 2015

Sociedad Estatal SEGITTUR. (2013). Segittur Turismo e Innovación. Recuperado el 22 de Mayo de 2016, de Estudio de Mercado de Apps Turísticas: Obtenido: http://www.think-med.es/wpcontent/uploads/group-documents/5/1386234639-Segittur_APPS-Turismo.pdf

Sulser, R. A., \& Pedroza, J. E. (2004). Exportación Efectiva. México: Ediciones Fiscales ISEF.

Toro, J., \& Hurtado, I. (2007). Paradigmas Y Metodos de Investigacion en Tiempos de Cambios. Caracas: Ediciones CEC. S.A. 